\title{
Belphégor
}

\section{Petit bord sombre à l'horizon de la pensée...}

Entretien avec Charles Grivel

\section{Marc Lits and Charles Grivel}

\section{(2) OpenEdition}

Journals

Electronic version

URL: https://journals.openedition.org/belphegor/1366

DOI: 10.4000/belphegor.1366

ISSN: 1499-7185

Publisher

LPCM

Electronic reference

Marc Lits and Charles Grivel, "Petit bord sombre à l'horizon de la pensée...", Belphégor [Online], 16-1 |

2018, Online since 27 March 2019, connection on 19 October 2021. URL: http://

journals.openedition.org/belphegor/1366 ; DOl: https://doi.org/10.4000/belphegor.1366

This text was automatically generated on 19 October 2021.

\section{(c)}

Belphégor est mis à disposition selon les termes de la Licence Creative Commons Attribution - Pas d'Utilisation Commerciale - Pas de Modification 4.0 International. 


\title{
Petit bord sombre à l'horizon de la pensée...
}

\author{
Entretien avec Charles Grivel
}

Marc Lits and Charles Grivel

\section{REFERENCES}

Charles Grivel, « Petit bord sombre à l'horizon de la pensée... », Textyles [En ligne], 10 | 1993, mis en ligne le 09 octobre 2012, consulté le 07 janvier 2019. URL : http:// journals.openedition.org/textyles/1893; DOI : 10.4000/textyles.1893.

1 Textvles - Le Fantastique peut-il être compris comme une question de regard, de point de vue?

2 Charles Grivel - Je le dis dans mon livre ${ }^{1}$, je le crois. Un œil se rencontre lui-même dans les émanations qu'il exécute. Il s'y plait, il s'y déplait aussi. Il y reconnaît. Il en redemande. Vous dites "perspective»? Moins. L'œil ne se définit pas par la perspective: il est large, trop large, il aimerait un peu plus des œillères: la "perspective » est une invention de narratologue. Pour moi, les mots fusent, les mots constellent le mental, ils partent un peu dans toutes les directions. Et surtout : ils sont visibles; je rencontre mon esprit dans les mots à leur vue. Ceci est une façon un peu compliquée de dire que je m'applique, non seulement à déchiffrer en lisant, mais à regarder. Ce n'est pas une chose qui est admise, je le sais bien. Mon éditeur n'a toujours pas compris, question financière mise à part, qu'écrire est dans la vue et qu'il fallait montrer cela par les documents appropriés que j'avais rassemblés, dommage. La vue est mise aux choses. Les choses sont mises en vue. La difficulté de regarder - de voir ce qu'on regarderait un peu plus - est, pour l'objet qui nous concerne, fondatrice. Le Fantastique participant en effet autant de la dérobade que de la monstration. Il est même exactement voué à cela. C'est pour cela aussi qu'il est moderne.

Le Fantastique est-il une littérature de simulacre, ou un simulacre de littérature?

4 Une opération. Ouvrir, à l'aide de mots (de descriptions de mots), puis fermer ; ouvrir en maintenant fermé ; fermer en suspendant. Ce n'est pas le savoir qui agit, mais l'œil. 
Ou c'est alors un savoir aussi bien conscient qu'inconscient. Le Fantastique est une opération d'écriture. donc apte à venir s'y déposer - d'ailleurs, il n'y a pas d' objet non plus, mais cela qu'on dit qu'il pourrait être - des mots accompagnés de leur évanescence, et puis du voir. Il ne faut pas se hâter de donner un statut à ce qu'on lit. Pour qu'une chose apparaisse, là où elle ne devrait pas, et donc se fasse craindre, il lui faut nécessairement un fond - un décalage, une différence, un abime : que le regard soit à la fois tendu et absorbé, fasciné et révulsé. Que les puits, les gorges - profondes et féminines -, les ravins, les ravines, les cavités, les souterrains, les trous, pullulent dans les récits fantastiques ne signifie pas qu'il y a du lieu, mais plutôt que le texte pointe sa béance l'esprit du texte - notre esprit dans le texte.

Réduire ? Ce n'est pas moi qui le ferais ! La littérature populaire est basique : c'est d'elle que tout dépend. Maintenant, oui, les gens aiment ça: avoir peur, regarder dedans ce qui leur échappe. Il faut les comprendre : on leur dit à longueur de journée sur le petit écran comment il faut faire (pour avoir, pouvoir et plaire) et ils constatent à longueur de journée aussi que la recette ne marche pas. Alors ils pensent. Ils pensent en lisant. Ils pensent en regardant au fond des mots les pistes qu'on leur donne. Ils se perdent en grand nombre, certes, avec délices. Je fais partie du grand nombre.

Vous lâchez là la grosse question! La question qui, comme nous voyons, anesthésie toute autre. Il faut savoir d'abord ce qu'est un genre. Du point de vue de l'histoire littéraire, disons que c'est une commode étiquette, une façon didactique de mettre les œuvres en des catégories, je ne suis pas sûr que cela apporte tellement. Du point de vue de la théorie de la communication, on pourrait défendre l'idée qu'un genre balise une façon de comprendre et que le lecteur obtempère. Mais est-ce si vrai ? Faisons-nous à ce point, dans nos pratiques nocturnes au moins, de si parfaites distinctions? Il est avéré, pour tous les bibliothécaires et pour tous les libraires, que la littérature narrative - et l'autre aussi ! - est « fiction » et nous supportons patiemment (?) cette exclusion. Je ne crois pas qu'il nous importe, comme lecteur, de savoir si telle ou telle chose qu'on nous raconte est vraie ou si elle ne l'est pas: cette... inquiétude est seconde. Par contre, il nous importe de la subir. C'est-à-dire de participer à sa monstration (sous barre, tant qu'on voudra). J'ai trouvé ce mécanisme plus important à décrire. Je n'ai pas voulu élaborer de fichier.

Le Fantastique est-il une littérature du refus?

Le Fantastique est une littérature du réel - acceptons pour un instant le mot - en tant qu'il manque. Une chose me frappe, c'est que l'imaginaire est dans le réel et que le réel terriblement, intolérablement, est en défaut. Je ne vois ni outre-lieu, ni outretombe, nous voici assis directement dans le trou, à table en quelque façon avec nos vampires et nos fantômes, nous sommes nous-mêmes ces monstres que nous convoquons, qui nous sont, on le serait à moins !, hospitaliers, nous-mêmes rions de leurs rires, nous-mêmes plantons leurs canines. Je ne vois pas là matière à un refus, mais au contraire une soif. Celui qui parle et celui qui lit aussi a soif; son déficit est de ne pas être assez là, en prise, présent, identifié, contaminé, je me souviens, un soir, en forêt de Compiègne, 
être tombé en panne; je m'étais rangé sur le bas-côté en surveillant les moindres bruits; ma lampe de poche refusait obstinément de marcher. Entre minuit et une heure, beaucoup plus tard, sur cette route parfaitement désertée, un automobiliste vint à s'arrêter. Il nous remorqua, il nous mena chez lui. Quand nous arrivâmes, il nous dit que la maison venait de brûler. Et nous dormîmes, exténués, cependant dans les cendres. Je crois qu'il ne s'agit que de cela: rencontrer, nous étions satisfaits de rencontrer. Pour cette raison, notre sommeil fut profond. J'oublie de dire que M. Martin - oui, Martin -, notre sauveur, était propriétaire d'un magasin d'articles de pêche et qu'il gagnait sa vie en vendant, parallèlement ( ?), de la pellicule. Notre couche sentait le brûlé. Nous nous tenions sagement au milieu de la photographie.

14 J'ai dit dans mon livre qu'il n'y a, d'une certaine manière, que cela. L'écriture des modernes est virtuellement fantastique.

Fantastique en ce que le principe de réalité est, depuis la Révolution de 89, je veux dire : depuis la révolution industrielle, atteint. En son principe atteint. L'ère de la consommation impose la production et ruine ce qui a toujours été là. À partir du moment où la valeur des objets dépend de leur degré de désirabilité et que celui-ci devient consciemment manipulable, alors il y a place pour le Fantastique. J'ai dit, haut et fort, que le Fantastique est le genre par excellence moderne. Son pendant est le policier. J'ai dit aussi - pour les mêmes raisons - qu'il était postmoderne. Toute écriture narrative emprunte désormais, peu ou prou, ce chemin : d'avoir à montrer ce qui est, ce qui ne parvient pas à être, le problème n'est pas le monstre ou la maison fendue par le milieu et qui branle, mais que nous soyons impuissants à les faire arriver, comme ils sont, parmi nous.

Peut-il y avoir du Fantastique sans récit?

16 Je viens d'apporter la preuve du contraire : dès que ça raconte, le Fantastique est de la partie. Si la peur monte. Si l'interférence est forte entre ce que j'accepte de regarder et ce que je refuse de considérer, le récit est un système de relais, de délais, de reports ; il creuse bien dans l'attente. La poésie, au contraire, fonctionne sans suspense, peindre signifie montrer, mais l'immédiateté de la toile (ou de ce qui en tient lieu) est telle qu'il est difficile d'y inclure, pour le regard qui s'en saisit, les sas nécessaires. Le récit bloque voir. Distille un mot à mot subtil dans l'âme. Qui vois-je que je ne puis voir en peinture?

17 Le Fantastique appelle-t-il nécessairement l'outrance?

18 Il faut s'entendre. Un récit sans outrance, c'est-à-dire sans excès dans le mode de représentation (la représentation est toujours un mode), n'attire guère, quand il n'ennuie pas. Or, le Fantastique suppose l'effroi et l'effroi ne s'obtient pas avec de petites mesures. Vous pouvez bien, comme Peter Roth, faire d'un homme un sein, ou comme Marcel Béalu, un œuf. Mais le hic est que vous puissiez mettre votre lecteur dans cette peau-là si j'ose dire - avec angoisse. Vous pouvez vous contenter de faire penser et de faire rire - comme le premier. Mais le second n'est pas loin de vous faire accéder, vous lecteur, à ce que vous n'êtes pas: Marcel Béalu aurait mis un peu plus d'outrance, il aurait étiré un peu plus son récit dans le temps qu'il aurait réussi un assez beau coup fantastique, oui, je pense. 
(1) et marqué, ingéré et expulsé. C'est ce que tentait d'expliquer mon analyse du Cœur mangé - conte évidemment biographique. Mais la découpe empreint toute corporalité. Regardons un instant les Anatomies de la fiancée, de Max Ernst, le pied sur le livre, de Bataille avec le même peintre, la reproduction fait foi. Regardons encore saillir tous ces morceaux du tissu convulsivement serré, puis ouvert, illustration populaire, j'en conviens, et nonobstant véridique : tout indique le un et non pas lui, qu'il se ressemble mais se désassemble. Considérons le coup qui lui est porté, en plein dans l'unité symbolique, en plein sexe en somme. Le Fantastique emmagasine ces effets-là. Vers l'unique. Vers le pire. Depuis deux siècles, nous savons que nous n'avons plus que cette 
forme-là : nous nous l'ôtons, nous nous la remettons, alternativement, «fantastiquement ».

Venons-en au Fantastique belge. Jean Ray semble, pour vous, offrir le prototype du récit fantastique. Pourquoi?

Il écrit dans le noir. Mais pourquoi m'agrafer une exclusive? Une préférence n'est pas une exclusive.

8 Le Fantastique n'a-t-il pas connu le succès qu 'on sait en Belgique parce que ce pays n 'arrive pas à se trouver une identité culturelle, géographique ou historique? Est-ce là le reflet de sa nonhistoire?

- à supposer que cet individu existe - en rajoute en effet sur ce qu'il n'est pas, le Suisse aussi, le Français de même. Baudelaire indiquait que les balcons de Bruxelles lui paraissaient dépourvus de sens, puisqu'il n'y avait selon lui rien sur la chaussée qui valait d'être regardé. Mais Tartarin qui gravit le Rigi par la mauvaise pente lui aussi s'assure en plein brouillard de contempler, de la terrasse de l'hôtel, le plus sublime spectacle. Qui a raison? Celui qui voit ou celui qui ne regarde pas? Quant à l'identité dont vous parlez, je ne crois pas que c'est le point: quand les gens commencent à se poser ce genre de question, il faut Sarajevo. Et Sarajevo donne plutôt dans le genre engagé que dans le genre fantastique! Mais on peut dire, en effet, que pour l'imaginaire, l'un vaut l'autre.

Malpertuis est-il un texte canonique du Fantastique?

31 Un trou qui n'est pas le bon trou fait rêver : Renard habite là et vous savez qu'il y fit s'enfoncer, par la tête, le loup et qu'il se trompa résolument à propos de la partie qui en ressortait et qu'il en fit usage... Nous nous trouvons dans le même cas : il y a un dessous et un dessus, un dedans et un dehors, un moi et un toi, l'excellent orifice et l'infâme orifice, un lieu et un non lieu. Le texte fantastique distribue cette donne. Je trouve que le roman de Jean Ray joue bien. Rappelez-vous la scène dans le magasin. Écoutez l'odeur. Entendez la pénombre. Touchez la double personne familière qui se rapproche silencieusement de vous. Canonique? En tout cas, inimitable.

Rangez-vous Magritte dans la case fantastique ou dans le merveilleux?

Ici ou là, je doute qu'il le faille, talent mis à part, je trouve que c'est un fantaisiste accru : une pipe est une pipe avant de n'en être pas une, ne l'oublions pas!

Le Fantastique belge, après Jean Ray, choisit plutôt la voie du glissement imperceptible, du décalage léger, $n$ 'est-ce pas contradictoire avec l'idée du Fantastique comme outrance? Pensons à Owen.

Il faut savoir que la légèreté est grave : les anges aussi volent bas. D'abord, le glissement dont vous parlez n'est pas imperceptible, il n'y aurait autrement pas lieu d'en parler. Ensuite, le décalage en question est agi par toutes sortes d'êtres semi-présents, semibêtes et semi-femmes, et il se fait craindre : en effet, ce choix est redoutable. Enfin, en telle matière, je ne crois pas au progrès. Quand votre auteur écrit - début de La cave aux crapauds : "Il faisait terriblement calme et chaud. L'air paraissait vibrer au-dessus du buisson de l'autre côté de la route ", à mon sens tout est dit : et ce qui va sortir du textuel et ce qui va sortir du végétal! ce sera une " outrance », oui, d'une assez bonne sorte. Pas plus intime que l'autre.

Y a-t-il, en peinture, dans le surréalisme, un courant fantastique? 
37 La peinture fantastique est une gageure, puisqu'il est particulièrement malaisé de montrer sans pourtant montrer sur une toile qui s'adresse à notre perception oculaire. Par ailleurs, je tiens que le Fantastique est lié à la production de l'effroi. Cela suppose le développement linéaire et l'extension dans le temps. Certes, le regard peut être guidé lorsqu'il est porté sur la toile par les objets mêmes qu'il rencontre ; cependant, son premier mouvement est celui de l'embrassement simultané et anéantit tout suspense. Il manque le temps à la toile. Ses objets peuvent être découpés, dérobés, restreints : ils se font pourtant voir pour ce qu'ils sont dans l'instant de la perception. Or, le texte distend mentalement cela. Le texte ou le récit filmique. Quant au surréalisme, ne confondons pas : il désire plaire et non point effrayer, l'étonnement qu'il élabore ne concerne pas la poussée que j'ai voulu considérer. L'inexistence des propositions n'est pas un critère de vérité de ces propositions ; il faut s'habituer à cela.

\section{NOTES}

1. Fantastique-Fiction. Paris, P.U.F., 1992, 256 p., coll. « Ecriture ».

2. Ch. Grivel est le Président de l'Association des Amis du roman populaire (NdlE). 